\title{
O sistema de enfermagem hospitalar: visualizando o cenário das políticas gerenciais ${ }^{1}$
}

The system of hospital nursing: visualizing the scene of the managerial politics

\author{
EI sistema del oficio de enfermera del hospital: visualizar la escena de la política gerencial
}

\author{
Maria Anice da Silva', Alacoque Lorenzini Erdmann", Renata da Silva Cardoso"'I
}

\section{RESUMO}

A concepção de sistema de enfermagem pode ser estruturada apoiada pelos fundamentos teóricos da ciência da complexidade nos seus processos interativos e relacionais. Assim, objetivou-se conceber a estrutura do processo auto-organizador do Sistema da Enfermagem hospitalar, pelo olhar da complexidade, visualizando as políticas gerenciais e suas interrelações na produção de serviços de Enfermagem. Trata-se de um estudo teórico conceptivo e reflexivo analítico sobre os conteúdos desenvolvidos no ensino de administração no curso de graduação em enfermagem nos cenários da prática gerencial. O processo auto-organizador possibilitou visualizar os componentes e as inter-relações na produção de serviços de enfermagem. Esta produção de serviços é resultante de uma dinâmica de inter-relações entre os sistemas num processo continuo de autonomia, interdependência e dependência. Todo esse processo é gerenciado pelo enfermeiro e centrado nas necessidades do cliente, mostrando-se importante para a vida e para a sobrevivência organizacional do sistema de enfermagem hospitalar.

Palavras chave: Enfermagem; Organização e Administração; Administração Hospitalar.

\section{ABSTRACT}

The conception of nursing system can be structuralized supported by the science theoretical basis of the complexity in its interactive and relational processes. Thus, it was objectified to conceive the structure of the self-organizing process of the Hospital Nursing System, for the look of the complexity, visualizing the managerial politics and its Interrelations in the production of services of Nursing. One is about analytical conceiving and reflective study theoretical on the contents developed in the education of administration in the degree course in nursing in the scenes of practice the managerial one. The self-organizing process made possible to visualize the components and the Inter-relations in the production of nursing services. This production of services is the result of Inter-relations dynamics between the systems in an extended process autonomy, interdependence and dependence. The whole process is managed by the nurse and centered in the necessities of the customer, revealing its importance for the life and the organizational survival of the hospital nursing system.

Key words: Nursing; Organization and administration; Hospital Administration.

\section{RESUMEN}

El concepto del sistema del oficio de enfermera puede ser estructurada apoyado por los lechos teóricos de la ciencia de la complejidad en sus procesos interactivos y emparentados. Así, era objetivo concebir la estructura del proceso autoorganizador del sistema del oficio de enfermera del hospital, para la mirada de la complejidad, visualizando la política gerencial y sus interrelaciones en la producción de servicios del oficio de enfermera. És un estudio teórico conceptivo y reflexivo analítico sobre los contenidos desarrollado en la educación de la administración en el curso de la graduación en el oficio de enfermera en las escenas de práctico gerencial. El proceso auto-organizador hizo posible visualizar los componentes y las interrelaciones en la producción de los servicios del oficio de enfermera. Esta producción de servicios es resultado de una dinámica de interrelaciones entre los sistemas en un proceso

\footnotetext{
Artigo produzido a partir da Tese: Silva, M A. Complexidade e Sistema de Enfermagem Hospitalar. 2006 Tese (Doutorado em Engenharia de Produção) - PG em Engenharia de Produção - ÁC de Ergonomia, da UFSC e das produções do GEPADES - Grupo de Estudos e Pesquisas em Administração em Enfermagem e Saúde - Dpto. de Enf/CCS/UFSC.

'Profa da UFSC, Dra. Enf., Subcoordenadora do GEPADES, maria_anice@hotmail.com.

"Profa. da UFSC, Dra. Enf., pesquisadora do CNPq, Coordenadora do GEPADES, alacoque@newsite.com.br

III Mestranda da UFSC, Enf. Membro do GEPADES, renatadasilvacardoso@hotmail.com
} 
Silva MA, Erdmann AL, Cardoso RS. O sistema de enfermagem hospitalar: visualizando o cenário das políticas gerenciais. Revista Eletrônica de Enfermagem [Internet]. 2008;10(2):448-459. Available from: http://www. fen.ufg. br/revista/v10/n2/v10n2a16.htm

continúa de la autonomía, de la interdependencia y de la dependencia. Todo este proceso es manejado por la enfermera y centrado en las necesidades del cliente, de revelar importante para la vida y de la

\section{NTRODUÇÃO}

O hospital é reconhecido como uma das mais complexas organizações já concebidas, investindo no avanço tecnológico em saúde e na capacidade de atender à emergência de novos e diferentes problemas de saúde da sociedade.

Assim, as práticas gerenciais do cuidado de Enfermagem hospitalar necessitam de avanços tecnológicos mesmo reconhecendo-se a competência dos enfermeiros nas atividades organizativas, talvez, decorrentes do seu exercício intenso de mobilização da operatividade do Sistema de Enfermagem.

As estruturas organizativas da Enfermagem hospitalar vêm se mostrando eficientes na produção do cuidado à saúde dos clientes internados nas instituições hospitalares, porém pouco explicita a descrição ou clareza do processo de produção nas práticas das atividades e situações do cotidiano administrativo da Enfermagem ${ }^{(1)}$.

As atividades administrativas são inerentes e fundamentais no processo produtivo do Sistema de Enfermagem. Em outras palavras, cuida-se administrando ou gerenciando e administra-se ou gerencia-se cuidando. São processos e contra-processos interdependentes cuja competência da profissão de Enfermagem se sustenta por uma forte exigência de conhecimentos sobre o processo produtivo gerencial.

A ciência da complexidade vem sendo utilizada para dar respostas a questionamentos de ordem das interatividades, conexões, pluralidade, diversidades, complementaridade, contradições, incertezas, dentre outros, que caracterizam os fenômenos sociais no cotidiano das organizações sociais. Assim, a compreensão dos movimentos nas organizações pode ser sustentada pelos conhecimentos sobre Sistemas Adaptativos Complexos.
Esses sistemas
demonstram

características identificáveis: enraizamento, a supervivencia organizacional del sistema del oficio de enfermera del hospital.

Palabras clave: Oficio de enfermera; Organización y administración; Administración Hospitalaria.

auto-organização,

não-linearidade, imprevisibilidade, e outros. Apresentam comportamento emergente que surge a partir de regras simples e interligações entre os diversos elementos com fronteiras porosas e, como eles interagem respondem ao ambiente. Os sistemas de saúde e da profissão de enfermagem podem ser vistos como sistemas complexos adaptativos e, quando isso ocorre, nova visão pode ser adquirida ${ }^{(2)}$.

Estudo realizado(3) visando focalizar os sistemas organizacionais da Enfermagem nas instituições de saúde diante da noção de complexidade pela auto-organização, constata que as redes dos fenômenos organizacionais sobrevivem, se modificam e se mantém pelos múltiplos e complexos canais de relações, que nem sempre podem ser 'administráveis'. As relações multidimensionais com os diferentes meios dos sistemas estão em constantes movimentos frente a influências múltiplas e imprevisíveis.

No ensino de Administração em Enfermagem tem-se a preocupação e a responsabilidade de debater e construir com o futuro profissional, novos referenciais apoiados por novas tecnologias gerenciais, a fim de torná-lo um agente de mudança da realidade dos Sistemas de Enfermagem das instituições de saúde, bem como, de constante busca de um espaço que propicie um melhor viver e conviver no mundo do trabalho. Nesse sentido, para se avançar na proposição de tecnologias gerenciais na Enfermagem, é importante partir para a busca de melhor clareza e sustentação teórica dos seus processos produtivos, apoiados por referenciais teóricos atuais e consistentes.

Trata-se de uma proposta de descrição dos Sistemas de Enfermagem sob o enfoque de sistema complexo, orientado por alguns referenciais teóricos de sistemas de produção de serviços e sua viabilidade como sistema auto-organizador, que facilite compreender e 
Silva MA, Erdmann AL, Cardoso RS. O sistema de enfermagem hospitalar: visualizando o cenário das políticas gerenciais. Revista Eletrônica de Enfermagem [Internet]. 2008;10(2):448-459. Available from: http://www.fen.ufg. br/revista/v10/n2/v10n2a16.htm

lidar com as conexões, contradições, diversidades e incertezas.

Assim, questiona-se: que concepção de sistema de enfermagem hospitalar pode-se chegar ao caracterizar o processo autoorganizador do Sistema da Enfermagem hospitalar, pelo referencial do paradigma da complexidade, visualizando as políticas gerenciais e suas inter-relações na produção de serviços de Enfermagem?

Parte-se de alguns pressupostos elaborados a partir da compreensão dos conteúdos teóricos do paradigma da complexidade e do domínio e vivência das autoras sobre a gerencia ou administração de enfermagem e saúde. Estes pressupostos orientam a construção dos conhecimentos pretendidos neste estudo. São eles:

O Sistema de Enfermagem é o lócus da produção de serviços relativos ao cuidado, envolvendo as dimensões tecnológicas, gerenciais, éticas e educativas, possui relações múltiplas de dependência, interdependência e de autonomia que se configuram numa estrutura dinâmica complexa.

O Sistema de Enfermagem produz serviços como resultado de uma dinâmica de inter-relações entre os demais sistemas hospitalares num processo contínuo de autonomia, interdependência e dependência. Todo esse processo desenvolve-se centrado nas necessidades do ser humano cliente.

A organização do trabalho da Enfermagem se sustenta por políticas gerenciais instituídas e consolidadas na prática administrativa hospitalar, que podem ser visualizadas no seu processo auto-organizador.

Os processos autoorganizadores/adaptativos da Enfermagem estão voltados para os produtos do cuidado, numa dinâmica contínua de conhecimentos e ações interdependentes, orientadas por regras (normas, rotinas) definidas, que se efetivam na flexibilidade da ordem e desordem do acaso.

O Sistema de Enfermagem mobiliza-se pela atuação do enfermeiro, líder da equipe, que proporciona os meios adequados para que os sujeitos agregados ao sistema possam exercitar sua autonomia o suficiente para aprender. E, por meio do aprendizado e da adaptação às novas situações, colaborem uns com os outros e consigam auto-organizar-se produzindo serviços coerentes com os objetivos do próprio sistema e da instituição de saúde.

Apoiados por esses pressupostos, têm-se como objetivo deste estudo, caracterizar o processo auto-organizador do Sistema da Enfermagem hospitalar, pelo referencial do paradigma da complexidade, visualizando as políticas gerenciais e suas inter-relações na produção de serviços de Enfermagem.

\section{MÉTODOS}

Trata-se de um estudo teórico conceptivo e descritivo sobre a estrutura organizacional do Sistema de Enfermagem hospitalar. Foi delineado a partir dos conteúdos desenvolvidos no ensino de administração no curso de graduação em enfermagem, nas informações documentais dos hospitais da região onde são realizadas as atividades de ensino prático e na experiência profissional dos pesquisadores, fundamentado nos conceitos da teoria da complexidade. O estudo foi realizado através do Grupo de Estudos e Pesquisas em Administração de Enfermagem e Saúde (GEPADES), em Florianópolis - Santa Catarina - Brasil, no período de Junho de 2004 a Julho de 2006.

Como fonte de dados para a descrição, reflexão e construção da estrutura do Sistema da Enfermagem hospitalar, visualizando as inter-relações na produção de serviços, foram utilizados documentos formais e procedimentos informais da gestão dos serviços de enfermagem hospitalar, bem como os textos e literatura do ensino de Administração em Enfermagem nos cursos de graduação e especialização em enfermagem e de administração hospitalar da UFSC, além das fontes de referência em administração hospitalar.

A concepção da estrutura partiu da análise dos modelos organizacionais da enfermagem até então aplicados, fundamentando-se no referencial teórico selecionado. Teve como base também a análise das inter-relações das políticas de gestão na produção dos serviços de enfermagem com os demais sistemas do hospital, visualizando a autonomia, interdependência e dependência com estes 
Silva MA, Erdmann AL, Cardoso RS. O sistema de enfermagem hospitalar: visualizando o cenário das políticas gerenciais. Revista Eletrônica de Enfermagem [Internet]. 2008;10(2):448-459. Available from: http://www.fen.ufg. br/revista/v10/n2/v10n2a16.htm

sistemas. Na seqüência, com o resultado da análise do processo auto-organizador, pode-se partir para a concepção da estrutura do processo auto-organizador na produção de serviços de enfermagem, utilizando-se de imagens gráficas para representar estas estruturas e interações.

A fusão das políticas de gestão no processo de produção de cuidado, permeada pelas variáveis do processo auto-organizador formando uma rede de conexões e de relacionamentos, foi o resultado da estrutura concebida.

O procedimento de análise e construção do conhecimento acerca da estrutura organizativa da Enfermagem foi um processo reflexivo, flexível, com idas e vindas, na qual se procurou percorrer todos os caminhos da administração, do ensino de administração e os desafios da teoria da complexidade.

Para certificação da credibilidade das estruturas concebidas, na medida em que o estudo foi sendo realizado e representado, alguns procedimentos de validação foram adotados, tais como: validação aparente, validação de conteúdo, validação comparativa, validação com expert e validação de aplicação, que permitiram avaliar e aperfeiçoar os modelos a cada etapa de sua elaboração.

A representação da estrutura concebida foi submetida à validação por comparação por um grupo de 06 enfermeiros chefes de serviço e professores de administração em enfermagem. O grupo reuniu-se em dois momentos: no primeiro a organização do Sistema de Enfermagem foi apresentada no modelo clássico; no segundo momento, a organização do Sistema foi apresentada no modelo concebido.

Após as duas apresentações o grupo de enfermeiros fez a comparação dos modelos e a validação da estrutura proposta como a de melhor compreensão e que reúne o maior número de elementos necessários para identificar a realidade.

Realizada também a validação por 02 profissionais doutores e pesquisadores da área da saúde, com produção de conhecimentos na ciência da complexidade. A estrutura concebida foi apresentada e examinada com base nos conceitos oriundos da teoria da complexidade.

$\mathrm{Na}$ validação concluiu-se que a estrutura concebida contribuirá para identificar situações múltiplas já conhecidas, porém ainda não analisadas sob o olhar da complexidade e da auto-organização. Esta abordagem proporcionou uma nova compreensão da amplitude e dinâmica gerencial da Enfermagem hospitalar.

As inter-relações, até então representadas por fluxogramas, são visualizadas sob um novo paradigma de concepção, permitindo o refletir/repensar a prática gerencial da Enfermagem, principalmente no que se refere ao respeito à autonomia dos indivíduos e dos grupos, possibilitando o aprimoramento da qualidade dos serviços.

\section{DISCUSSÃO E RESULTADOS}

O Sistema de Enfermagem é definido pela Resolução do Conselho Federal de Enfermagem (4), como "conjunto de Unidades de Enfermagem que são constituídas pelos recursos físicos e humanos em uma instituição de assistência à saúde".

Os processos de atuação da enfermagem estão inseridos diretamente nos processos assistenciais do hospital, sendo considerado uma área de atividade-fim, pois suas ações estão relacionadas diretamente com as finalidades da instituição de saúde. Todavia, interage com os demais processos da instituição para produzir o efeito principal que é o cuidado, cuja qualidade ao final, depende da eficiência e da eficácia de cada um deles e da interação destes processos.

O Enfermeiro é o responsável técnico por este sistema e atua em equipe com os auxiliares de enfermagem, técnicos de enfermagem e auxiliares administrativos. Os profissionais da Enfermagem são os únicos profissionais dentro da instituição hospitalar, que atuam diretamente com o cliente, diuturnamente, num trabalho contínuo e integrado. Sua ação vai além do cuidado direto ao cliente, envolve ainda, a gerencia de todas as unidades assistenciais e afins da instituição hospitalar, tais como: Unidades de Internações, Centro Cirúrgico, Centro Obstétrico, Unidade de 
Silva MA, Erdmann AL, Cardoso RS. O sistema de enfermagem hospitalar: visualizando o cenário das políticas gerenciais. Revista Eletrônica de Enfermagem [Internet]. 2008;10(2):448-459. Available from: http://www.fen.ufg. br/revista/v10/n2/v10n2a16.htm

Neonatologia, Unidade de Terapia Intensiva, Emergência, Centro de Material e Esterilização, entre outros.

A produção dos serviços hospitalares sob o olhar da complexidade é resultante de uma dinâmica de inter-relações entre os sistemas e subsistemas, num processo contínuo de autonomia, interdependência e dependência. Todo este processo desenvolve-se centrado nas necessidades do cliente, onde o sistema de Enfermagem aparece contextualizado e interagindo com os outros sistemas e subsistemas, proporcionando uma rede de interconexões.

\section{A organização no Sistema de Enfermagem}

Os Sistemas de Enfermagem estão estruturados e organizados nas mais diversas instituições de saúde, sejam elas públicas ou privadas, de atendimento ambulatorial ou caracterizadas pela internação. É considerado como uma entidade social que como tal precisa ser organizado, levando em consideração as diretrizes básicas e princípios fundamentais de organização e de administração ${ }^{(5)}$.

Dependendo do tipo de hospital (público ou privado), o Sistema de Enfermagem detém 45 a $60 \%$ dos funcionários de todo o hospital e é responsável pela administração de todas as unidades prestadoras de cuidados do hospital, as quais são consideradas subsistemas do Sistema de Enfermagem ${ }^{(6)}$. Além da prestação do cuidado/assistência ao cliente, a enfermagem é responsável pelo provimento de pessoal, material e equipamentos, bem como, pelo processo assistencial e administrativo destas unidades.

O processo produtivo da enfermagem é dirigido por um enfermeiro subordinado diretamente ao diretor geral da instituição. Cabendo-Ihe, privativamente, "a direção do órgão de enfermagem integrante da estrutura básica da instituição de saúde, pública e privada, e chefias de serviço e de unidade de enfermagem", além da organização, planejamento coordenação, execução e avaliação dos serviços assistenciais de enfermagem ${ }^{(7,8)}$.

Essa conquista não somente garante legalmente a autonomia administrativa da
Enfermagem, como também prescreve o seu comprometimento e responsabilidade com a direção, planejamento, coordenação e organização do órgão de Enfermagem.

Assim, o Enfermeiro, considerado como um sistema de produção (isoladamente) fornece produtos, além de assistenciais (típicos da Enfermagem), as atividades gerenciais (planejamento, organização, direção e controle), investigativas, educativas e informacionais.

Um dos primeiros modelos organizacionais $^{(5)}$ para os Sistemas de Enfermagem propõe como integrantes gerais da organização: a filosofia do Serviço de Enfermagem, os objetivos, o regimento interno e as políticas de gestão, subdivididas em: estrutural-diretiva, de recursos humanos, de recursos materiais, de ensino, de pesquisa, assistencial e orçamentária.

Este modelo(5) já demonstrava a interdependência dos vários componentes da organização formal entre si, bem como a influência que cada componente pode receber dos demais ao mesmo tempo em que a exerce. "Ao passo que a inter-relação dos vários integrantes se dá de uma forma dinâmica e ininterrupta, o meio ambiente externo, da mesma forma, influencia e é influenciado pela organização"(5). A visão da autora ${ }^{(5)}$ já permitia visualizar a Enfermagem como um sistema auto-organizador, pois foi dada a ênfase na necessidade de preservar a autonomia dos indivíduos, seja ele cliente ou trabalhador.

O estudo do sistema de cuidados de enfermagem teve continuidade com a apresentação um esquema de fluxo estruturado $^{(3)}$ tendo como elementos: as fronteiras, objetivos, saídas e entradas, permeando as atividades básicas do sistema de produção. Neste estudo ${ }^{(3)}$, a autora identifica os problemas de um sistema de enfermagem explorando conceitos ou noções como: sensibilidade analítica, multiplicidade de fatos ou situações, inter-relações, estrutura, ordem, evento ou acaso, homogeneidade e heterogeneidade, ambíguo, incerto, impreciso, indeterminado, imprevisibilidade, dentre outros.

O cliente 'cuidado', os cuidados de enfermagem prestados ou a assistência de 
Silva MA, Erdmann AL, Cardoso RS. O sistema de enfermagem hospitalar: visualizando o cenário das políticas gerenciais. Revista Eletrônica de Enfermagem [Internet]. 2008; 10(2):448-459. Available from: http://www.fen.ufg.br/revista/v10/n2/v10n2a16.htm

enfermagem realizada são apontados como o produto do sistema de enfermagem. Este sistema visa prestar assistência para atender as necessidades de cuidados de saúde do cliente. O cliente vem buscar o tratamento e os cuidados que requerem como insumos: pessoal, material e informações, num processo de produção caracterizado pela prestação de serviços ${ }^{(6)}$. Portanto, o cuidado/assistência prestado é visto como o produto final do processo produtivo da enfermagem.

O sistema de cuidados de enfermagem pode extrapolar as fronteiras da instituição de saúde, assim como pode estar delimitado pelas fronteiras de uma unidade organizacional de enfermagem com finalidade própria. Também o sistema de cuidado de enfermagem pode conter o processo administrativo, como o sistema administrativo da enfermagem pode conter o seu processo de cuidado ${ }^{(9)}$.

Por outro lado, para se avançar na proposição de tecnologias gerenciais na Enfermagem é importante partir para a busca de melhor clareza e sustentação teórica dos seus processos produtivos apoiados por referenciais teóricos ${ }^{(10)}$. Nessa proposta as autoras fazem um ensaio da descrição do processo administrativo da enfermagem sob o enfoque de sistemas complexos, orientado por alguns referenciais teóricos de sistema de produção de serviços e sua viabilidade como sistema auto-organizador.

O processo de produção administrativa da enfermagem nas instituições hospitalares constitui-se num sistema dinâmico e complexo, servindo de cenário inesgotável de estudo para compreensão das estratégias de ação e dos processos organizativos.

A Enfermagem, como sistema de produção, tem produtos basicamente técnicoassistenciais, mas também, produz atividades técnico-administrativas e administrativas. $E$ produto em atividades administrativas é, igualmente, o resultado do sistema de produção do serviço. Estes produtos são os meios utilizados para prestação da assistência, os serviços burocráticos e os de apoio assistencial. Ou seja, há o ato intencional de produzir algo útil, que não é o cuidado de Enfermagem, mas Ihe dá suporte, como, preencher formulários de admissão ou prontuários, prestar informações, treinar a equipe, solicitar consertos, medicamentos e materiais, elaborar escalas de serviço e de férias, entre outros.

Hoje é possível perceber a preocupação da Enfermagem com a organização formal de seus Sistemas e visualizar as iniciativas adotadas na tentativa de organizar o trabalho, conquistar espaços dentro da organização hospitalar e delimitar a autoridade. No entanto, percebe-se também a preocupação em saber o que fazer para manter o Sistema organizado sem torná-lo excessivamente formal, burocrático e inflexível (11)

\section{Os Subsistemas do Sistema de Enfermagem}

Entre as unidades prestadoras de cuidados - Subsistemas do Sistema de Enfermagem - de um hospital geral de médio e grande porte, pode-se destacar: Emergência - adulto e pediátrica - (EA, EP); Unidades de Internação de Clínica Médica (UICM); Unidades de Internação de Clínica Cirúrgica (UICC); Unidade de Terapia Intensiva (UTI), adulto e pediátrica; Centro Cirúrgico (CC), adulto e pediátrico; Centro Obstétrico (CO); Unidade de Internação de Clínica Obstétrica (UICO); UTI Neonatal (NEO); Centro de Material e Esterilização (CME); Unidades de Internação de Clínica Pediátrica (UICP); Ambulatório, entre outras.

$\mathrm{Na}$ Figura 1, encontra-se a representação esquemática das inter-relações entre os subsistemas do Sistema de Enfermagem. As relações entre os subsistemas são inúmeras, criando um entrelaçamento de idas e vindas que se torna difícil representá-lo por meio de esquemas considerando a alta complexidade desse sistema. 
Silva MA, Erdmann AL, Cardoso RS. O sistema de enfermagem hospitalar: visualizando o cenário das políticas gerenciais. Revista Eletrônica de Enfermagem [Internet]. 2008;10(2):448-459. Available from: http://www.fen.ufg.br/revista/v10/n2/v10n2a16.htm

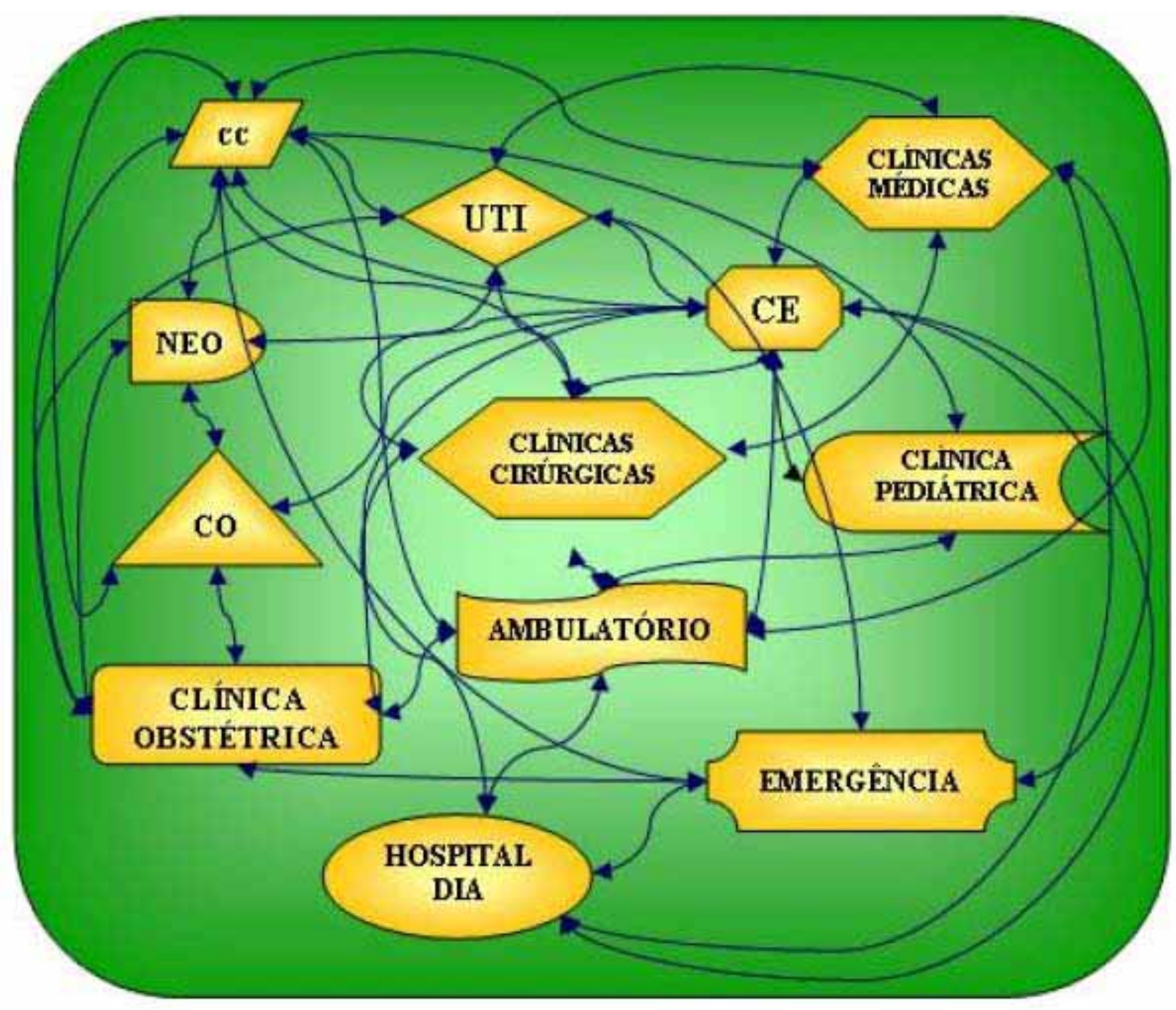

FONTE: Silva MA. Complexidade e Sistema de Enfermagem Hospitalar [tese].

[Florianópolis]: Universidade Federal de Santa Catarina; 2006

Figura 1: Representação esquemática das inter-relações entre os subsistemas do Sistema de Enfermagem, pelo processo auto-organizante.

Cada subsistema possui uma equipe de enfermagem composta por: Auxiliares e Técnicos de Enfermagem (profissionais de nível médio), Enfermeiros (profissionais de nível superior) e de Auxiliares Administrativos (profissionais de nível médio), os quais se agregam formando uma equipe de trabalho.

Outro aspecto a ser considerado é que, mesmo com todo este entrelaçamento nas relações entre os subsistemas, as características individuais de cada subsistema são preservadas existindo, neste cotidiano das relações, um "pacto" de respeito entre eles, principalmente no que se refere à autonomia no seu processo organizativo e gerencial.
Considerando que uma equipe, ou agregado, seja formada para a realização de propósitos organizacionais e que dentro de suas fronteiras os indivíduos atuam autonomamente, é interessante que cada um conheça os objetivos globais e a maneira pela qual espera que as funções de seu agregado venham a contribuir ${ }^{(12,13)}$. Se estes indivíduos participam do processo de definição destes objetivos, mais clara é a relação entre os objetivos e as metas de desempenho dos agregados ou equipes e, ainda, maior a disposição do indivíduo em deles fazer parte.

Da mesma forma, essa participação e visão global, por mais indireta que seja, permite 
Silva MA, Erdmann AL, Cardoso RS. O sistema de enfermagem hospitalar: visualizando o cenário das políticas gerenciais. Revista Eletrônica de Enfermagem [Internet]. 2008;10(2):448-459. Available from: http://www.fen.ufg. br/revista/v10/n2/v10n2a16.htm

maior domínio das restrições sofridas tanto pelo sistema em geral como pela própria equipe. Como conseqüência, "as regras que se deriva de tais restrições e que orientam a ação de cada indivíduo tornam-se mais inteligíveis"(9).

No entanto, não só a autonomia define o Sistema de Enfermagem como um sistema adaptativo complexo (SAC), mas $\operatorname{sim} a$ presença das variáveis do princípio da autoorganização no processo de produção.

O Sistema de Enfermagem com seus respectivos subsistemas estão organizados e estruturados em políticas de gestão (organizacional-diretiva, assistencial, pessoal, material, financeira e ensino e pesquisa) ${ }^{(5)}$. Para análise dos elementos das políticas de gestão do Sistema de Enfermagem, dentro do panorama dos SACs, optou-se pelas seguintes variáveis do processo auto-organizante: aprendizado, adaptação, agregação, autonomia, cooperação, inter-relação e auto-organização.

A intervenção de outros sistemas ou da direção superior ao sistema na construção desta política implica em cercear ou limitar a autonomia dos indivíduos, trazendo perdas das variáveis auto-organizantes. No entanto, há que se ressaltar, que a interdependência e a dependência de outros sistemas, da direção superior ou até mesmo do ambiente externo à organização, reforça as características do Sistema de Enfermagem como um Sistema Adaptativo Complexo(14).

\section{Concepção da estrutura de produção de serviços na enfermagem utilizando o processo auto-organizador}

Em toda a história da Enfermagem brasileira muito pouco se tem conhecimento a respeito de instituições de saúde que tenham criado obstáculos à organização do seu sistema de produção, principalmente no que se refere ao estabelecimento de princípios filosóficos, missão, visão, objetivos, metas, normas, rotinas e fluxo de trabalho.

O Sistema de Enfermagem hospitalar, de maneira geral, vem conquistando sua autonomia no processo reflexivo para elaboração e descrição dos seus instrumentos formais, sem que ocorra a intervenção de outros profissionais ou de instâncias superiores, inclusive com garantia conquistada em Lei Federal desde $1986^{(7)}$, como já foi discutido anteriormente. No entanto, é necessário que os profissionais adquiram conhecimentos e desenvolvam técnicas necessárias para condução de todo esse processo participativo da equipe de enfermagem.

Num processo coletivo de produção de serviços a informação e o aprendizado podem ser considerados como determinantes para a tomada de decisão e adaptação, pois "o sistema é capaz de ajustar seu comportamento a partir do que consegue perceber sobre as condições do seu meio ambiente e sobre seu desempenho" (15).

Como foi estudado até aqui, as políticas de gestão têm um papel fundamental como normas norteadoras das ações dos agentes agregados ao sistema. Porém, cabe salientar que o excesso ou a rigidez destas normas irá bloquear a autonomia dos indivíduos desfavorecendo o ambiente auto-organizador. A ação autônoma dos indivíduos e a interação são fundamentais para que estes indivíduos, por meio do aprendizado, da adaptação e da cooperação se auto-organizem.

$\mathrm{Na}$ produção dos serviços, as políticas de gestão, entendida como regras de comportamento, são previamente estabelecidas pelos agentes como estratégias de sobrevivência para as tarefas principais realizadas pelo sistema. Estas políticas estão inter-relacionadas de tal maneira que só é possível ser isoladas e representadas para fins didáticos, conforme foi realizado na análise do estudo. Entretanto, a linearidade das conexões não reflete a realidade do sistema com toda a diversidade de inter-relações e conexões entre as políticas de gestão, como também não retrata a inserção destas políticas na produção dos serviços.

Os agentes dos sistemas adaptativos complexos necessitam de ambiente adequado para desenvolver sua criatividade e proporcionar inovação. Isto nos remete a uma nova estrutura que permita a auto-reflexão e aprendizagem pela experiência, permitindo a ordem/desordem, a imprevisibilidade e a falta de controle ${ }^{(14)}$. 
Silva MA, Erdmann AL, Cardoso RS. O sistema de enfermagem hospitalar: visualizando o cenário das políticas gerenciais. Revista Eletrônica de Enfermagem [Internet]. 2008;10(2):448-459. Available from: http://www.fen.ufg.br/revista/v10/n2/v10n2a16.htm

Como toda a organização, nos sistemas adaptativos complexos pode ser observada uma interação contínua entre as suas redes informais e as estruturas formais (políticas de gestão). É neste momento que o conhecimento tácito é evidenciado e as políticas e procedimentos formais passam a ser filtrados e modificados pelas redes informais, permitindo que os agentes possam usar a criatividade quando se deparam com situações inesperadas $^{(14)}$.
Os processos de produção, na verdade, se confrontam com a ordem e desordem e com as certezas e incertezas, pois estes fatores são determinantes na emergência de adaptação, aprendizado e auto-organização. Conseqüentemente, isto proporciona uma constante adequação das políticas de gestão, gerando movimentos/ondulações constantes nestas políticas e entre elas. A Figura 2 representa, numa forma esquemática, estes movimentos e ondulações.

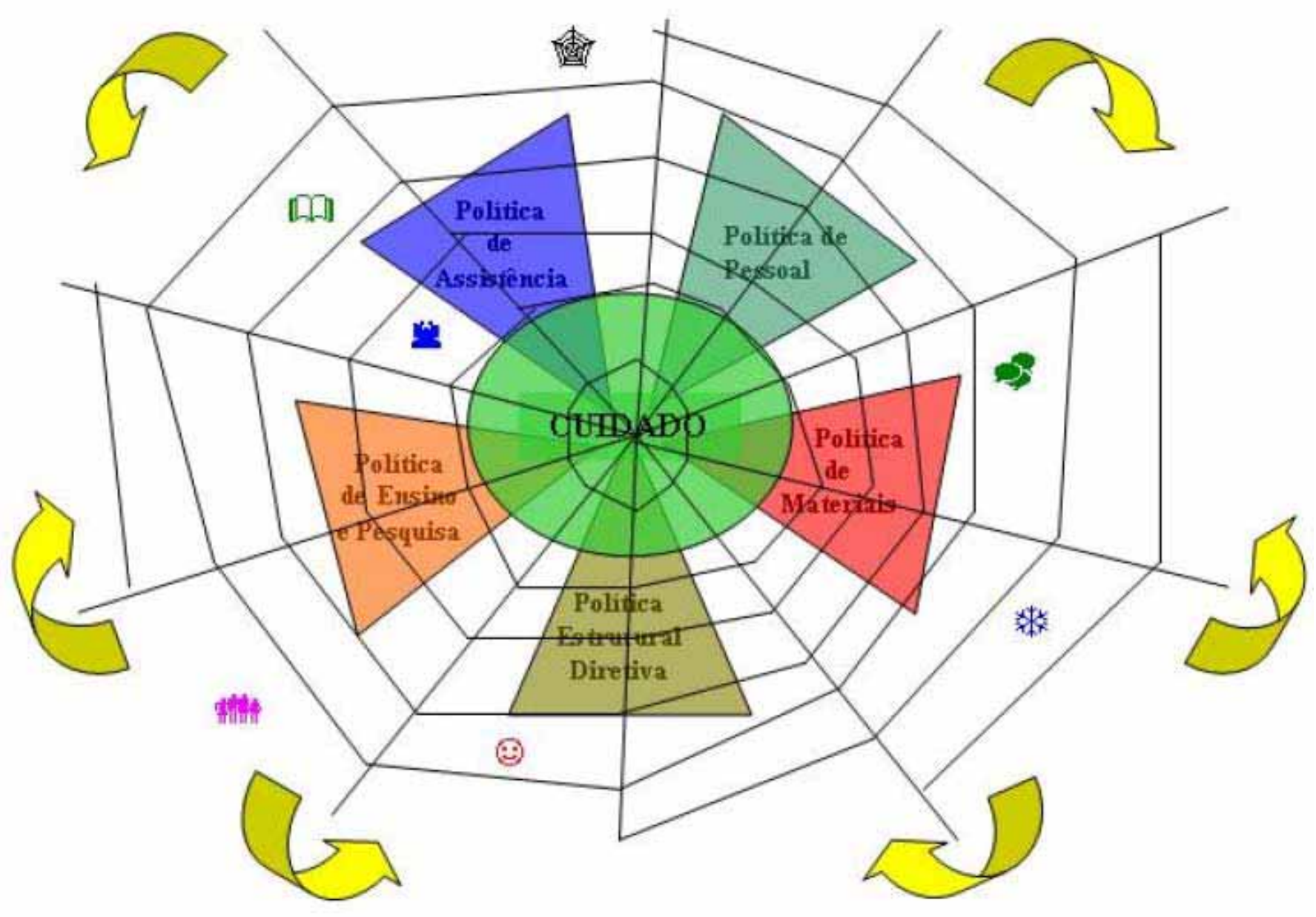

\begin{tabular}{|c|c|c|c|}
\hline Legenda: & D1 Aprendizado & * Adaptação & $\begin{array}{l}\text { (e) Cooperação } \Rightarrow \text { Inter-relação } \\
\text { Auto-organizacão }\end{array}$ \\
\hline
\end{tabular}

FONTE: Silva MA. Complexidade e Sistema de Enfermagem Hospitalar [tese].

[Florianópolis]: Universidade Federal de Santa Catarina; 2006

Figura 2: Estrutura representativa do processo auto-organizador na produção de serviços de enfermagem

O Sistema de Enfermagem tem como resultado da produção de serviço, o cuidado ao cliente. Este se processa por meio de procedimentos assistenciais e gerenciais que 
Silva MA, Erdmann AL, Cardoso RS. O sistema de enfermagem hospitalar: visualizando o cenário das políticas gerenciais. Revista Eletrônica de Enfermagem [Internet]. 2008;10(2):448-459. Available from: http://www. fen.ufg. br/revista/v10/n2/v10n2a16.htm

são estabelecidos pelas políticas de gestão. Estas políticas estão inseridas na dinâmica dos processos de produção de serviços, ora no cuidado direto ao cliente, ora na gerencia da unidade, e ainda, na interação com os demais sistemas do hospital e com o ambiente externo. Como sistema complexo, estes processos se desenvolvem dentro de uma estrutura autoorganizante.

A estrutura representada na Figura 2, resume e demonstra a estrutura autoorganizante das políticas de gestão do Sistema de Enfermagem, pela presença constante das variáveis do princípio auto-organizador: autonomia, aprendizado, adaptação, interrelação, cooperação, agregação e autoorganização.

Observa-se ainda na Figura 2, uma rede de inter-relações entre as políticas, criando vínculos permanentes de dependência e interdependência entre elas. O modelo concebe uma nova visualização da dinâmica gerencial do Serviço de Enfermagem.

No Sistema Adaptativo Complexo os agentes comportam-se de acordo com seus próprios princípios de interação local, num processo de auto-organização. Portanto, para que ocorra a auto-organização é necessária a existência de agentes interagindo localmente de acordo com seus próprios princípios (redes informais) e por meio de políticas por eles estabelecidas (redes formais), como foi analisado nos processos de gestão da Enfermagem, conforme constata os estudos de Chaffee MW, McNeill MM. ${ }^{(13)}$.

Esse sistema não gera anarquia, ele oferece as condições necessárias para que aconteça a criatividade e a inovação, não através de processos prévios, mas através do processo de auto-organização espontânea que produz resultados emergentes.

Pode-se afirmar que o sistema de Enfermagem é um Sistema Adaptativo Complexo, que aprende, evolui e interage com outros sistemas. Ele sobrevive porque aprende e evolui de maneira adaptativa, ou seja, registra informação para extrair regularidades, inserindo-a nas políticas de gestão que são continuamente mudadas à luz da experiência. Estão sempre em busca de padrões. Eles também interagem com o meio, aprendem com a experiência e se adaptam como resultado ${ }^{(14)}$.

Assim, como resultado da definição das políticas de gestão, pode-se constatar que a Enfermagem se auto-organiza não só no fortalecimento de abertura de canais de comunicação multidirecionais, como também, ao "ampliar a capacidade de percepção, interpretação e respostas a todos os tipos de feedback" internos e externos ao Sistema.

O papel do enfermeiro na liderança da equipe, desempenhado no modelo comandocontrole, não conseguirá dar conta da avalanche de mudanças que ocorrerão no ambiente dos SACs, pela presença do estímulo à criatividade no modelo auto-organizador. E neste caso, destaca-se que a importância do enfermeiro é o de criar e manter as condições propícias para que os indivíduos tenham o direito e sejam capazes de agir com autonomia, garantindo que suas ações estimulem e legitimem a participação e melhore a capacidade de perceber e compreender a sua realidade de trabalho ${ }^{(14)}$.

\section{CONSI DERAÇÕES FI NAIS}

O presente estudo possibilitou chegar à resposta da questão formulada acerca dos processos na dinâmica de inter-relação entre as políticas de gestão dos sistemas e subsistemas na produção de serviços de Enfermagem no contexto da organização hospitalar, sob o enfoque dos conceitos relativos à ciência da complexidade. Da mesma forma, os pressupostos foram evidenciados como pertinentes e adequados ao alcance dos objetivos.

Com a orientação destes pressupostos foram concebidas estruturas do processo autoorganizador do hospital e do Sistema da Enfermagem hospitalar, pelo referencial do paradigma da complexidade, visualizando as políticas gerenciais e suas inter-relações na produção de serviços de Enfermagem.

O sistema de Enfermagem constitui-se num processo auto-organizante das políticas de gestão do sistema, pela presença constante das variáveis do princípio auto-organizador: autonomia, aprendizado, adaptação, interrelação, cooperação, agregação e auto- 
Silva MA, Erdmann AL, Cardoso RS. O sistema de enfermagem hospitalar: visualizando o cenário das políticas gerenciais. Revista Eletrônica de Enfermagem [Internet]. 2008;10(2):448-459. Available from: http://www.fen.ufg. br/revista/v10/n2/v10n2a16.htm

organização. Este processo organizativo se estrutura numa rede de conexões e interrelações entre as políticas de pessoal, material, finanças, estrutura diretiva, e de ensino e pesquisa, dentre outras, criando vínculos permanentes de dependência e interdependência entre elas.

Analisando o Sistema de produção de serviços na Enfermagem sob a ótica da complexidade, pode-se afirmar que o processo de elaboração/construção dos instrumentos formais do Sistema permite a interação entre os profissionais da Enfermagem e de todo os sistemas e subsistemas do hospital, proporcionando a adaptação e aprendizagem, favorecendo a negociação e permitindo a autoorganização do Sistema. E, mesmo assim, o sistema permanecerá autônomo, já que o ambiente externo apenas desencadeia as mudanças, mas não as dirige ${ }^{(14)}$.

Desta maneira, o verdadeiro sentido do "papel" do enfermeiro, neste caso, passa para o de "tecer a rede" de inter-relações e estabelecer um contexto adequado ao emergir da criatividade e tomada de decisões, fazendo com que os profissionais da Enfermagem se voltem para um circuito comportamental de descoberta - escolha - ação(14).

$\mathrm{Na}$ Enfermagem o sistema sobrevive porque aprende ou evolui de maneira adaptativa, registrando informações para extrair as regras ou normas formais ou informais, inserindo-as nas políticas de gestão, que são continuamente mudadas à luz das experiências de seus agentes.

O modelo representativo proposto concebeu uma nova visualização da dinâmica gerencial do hospital e do sistema de enfermagem e possibilitou a construção de um novo referencial para a área específica da enfermagem, tanto no ensino, na assistência, como também na promoção de um novo olhar na competência gerencial do enfermeiro. $O$ estudo permitiu, ainda, a visibilidade da amplitude do papel do Enfermeiro, tanto na assistência quanto na administração da instituição hospitalar.

Cabe ressaltar, que no olhar da complexidade não se pode modelar um fenômeno em todas as suas dimensões, até mesmo por causa do próprio limite humano de percebê-lo totalmente, em suas abrangências e interferências. Por isso, a estrutura proposta não pretende contemplar o sistema de Enfermagem em toda a sua dimensão administrativa e organizacional. $\mathrm{O}$ que se apresenta continua sendo um desafio para o avanço da ciência da administração aplicada às organizações de saúde e da Enfermagem.

\section{REFERÊNCI AS}

1. Erdmann AL, Silva MA, Erdmann RE, Ribeiro JA. O reconhecimento do produto do sistema organizacional de cuidados de enfermagem. Ciência, Cuidado e Saúde. 2005; 4(1):37-46.

2. Schneider $M$, Somers $M$. Organizations as complex adaptive systems: implications of complexity theory for leadership research. Leadership Quarterly. 2006;4(17):351-65.

3. Erdmann AL. Sistema de cuidados de enfermagem. Pelotas: Universitária/UFPEL; 1996.

4. Conselho Federal de Enfermagem. Resolução COFEN no168/93. Baixa normas para Anotação da responsabilidade técnica de Enfermeiro (a), em virtude de Chefia de Serviço de Enfermagem,, nos estabelecimentos das instituições e empresas públicas, privadas e filantrópicas onde é realizada assistência à saúde. Rio de Janeiro (Brasil): COFEN; 1993.

5. Horr L. Modelo de organização do serviço de enfermagem. Rev. Gaúcha de Enfermagem. 1992; 13(2): 36-41.

6. Silva MA. Concepção ergonômica dos locais e dos espaços de trabalho de uma unidade de emergência hospitalar [dissertação]. [Florianópolis]:Programa de Pós-Graduação em Engenharia de Produção/UFSC; 1999.

7. Presidência da República (BR). Lei no 7.498 de 25 de Junho de 1986. Dispõe sobre a regulamentação do exercício da enfermagem e dá outras providências. Brasília: Diário Oficial da União; 1986.

8. Presidência da República (BR). Decreto $\mathrm{n}$ ㅇ 94.406 de 08 de Junho de 1987. Regulamenta a Lei no 7.498 de 25 de junho de 1986, que dispõe sobre o exercício da enfermagem, e dá outras providências. Brasília: Diário Oficial da União; 1987. 
Silva MA, Erdmann AL, Cardoso RS. O sistema de enfermagem hospitalar: visualizando o cenário das políticas gerenciais. Revista Eletrônica de Enfermagem [Internet]. 2008;10(2):448-459. Available from: http://www.fen.ufg. br/revista/v10/n2/v10n2a16.htm

9. Erdmann AL. Diagnóstico de problemas num sistema de enfermagem de hospital de ensino proposta de modelo [livre docência]. [Rio de Janeiro]: UERJ; 1987.

10. Silva MA, Erdmann AL, Cardoso RS. O processo de produção administrativa da enfermagem hospitalar: um sistema complexo viável. Revista Mineira de Enfermagem - REME. 2006; 10(1): 54-60.

11. Kurcgant P. Gerenciamento em enfermagem. Rio de Janeiro: Guanabara Koogan; 2005.

12. Agostinho ME. Administração complexa: revendo as bases científicas da administração. Revista Administração de Empresa - eletrônica [Internet]. 2003 [cited 2007 mar 06];1(2): 118. Available from: http://www.rae.com. br/ redirect.cfm?ID=1254.

13. Chafee MW, McNeill MM. A model of nursing as a complex adaptive system. Nurs. Outlook. 2007; 55(5): 232-241.

14. Silva MA. Complexidade e Sistema de Enfermagem Hospitalar [tese]. [Florianópolis]: Universidade Federal de Santa Catarina/UFSC; 2006. $195 \mathrm{p}$

15. Agostinho, ME. Complexidade e organizações: em busca da gestão autônoma. São Paulo: Atlas; 2003.

Artigo recebido em 22.05.07

Aprovado para publicação 30.06.08 